\title{
Assertive outreach for "difficult to engage" patients: a useful tool for a subgroup of patients in specialized early psychosis intervention programs.
}

Luis Alameda ${ }^{a b}$, Philippe Golay ${ }^{b c}$, Philipp Baumannab, Stéphane Morandic, Carina Ferrariab, Philippe Conus ${ }^{\mathrm{b}}$, Charles Bonsack ${ }^{\mathrm{c}}$

a Unit for Research in Schizophrenia, Center for Psychiatric Neuroscience, Department of Psychiatry, Lausanne University Hospital (CHUV), Switzerland

b Service of General Psychiatry, Treatment and Early Intervention in Psychosis

Program (TIPP-Lausanne), Lausanne University Hospital (CHUV), Switzerland

c Service of community Psychiatry, Department of Psychiatry, Lausanne University Hospital (CHUV), Switzerland

Corresponding Author: Luis Alameda, Department of psychiatry, LAUSANNE University Hospital (CHUV), Switzerland. Luis.alameda-fernandez@chuv.ch

\section{Introduction}

Over the last 20 years, many specialized early psychosis (EP) programs have been developed in Europe and around the world (Marshall and Rathbone, 2011), aiming at early detection and provision of phase specific treatment. One important challenge faced by clinicians involved in such programs is the prevention of patients' disengagement (Conus et al., 2010). The strategies applied to resolve this problem may vary between services, but case management (CM) interventions and assertive community treatment (ACT)(Marshall and Lockwood, 1998), which are essential ingredients of most programs (Marshall et al., 2004), are critical in this regard.

Case management has progressively developed during the last 30 years (Ziguras and Stuart, 2000), and the responsibility of clinicians assuming such positions in EP programs is to facilitate 
patient's engagement, conduct clinical assessment, plan treatment in collaboration with psychiatrists, facilitate linkage with available treatment resources, collaborate with families, and provide psychoeducation or crisis intervention (Lamb, 1980; Marion-Veyron et al., 2013). Similarly to ACT, CM was initially developed in the context of deinstitutionalization, where marginalized patients did not have access to local mental health centers and frequently were only treated in the frame of hospital admissions, often under pervasive measures (Mueser et al., 1998; Pepper et al., 2014). Original ACT is an evidence based intervention to address difficult to engage patients, which distinguishes itself from generic case management models by smaller case loads, 7/7 and 24/24 availability, almost exclusive community based intervention available up to several times per day (Morandi and Bonsack, 2011). Various forms of assertive outreach were developed in Europe, such as FACT in the Netherlands (Veldhuizen, 2007), or for early psychosis in Australia and Europe (Brewer et al.; Nordentoft, 2015), in order to fit different needs and mental health systems. In this article, we will refer to Intensive Case Management (ICM) rather than to ACT, considering our intervention (Bonsack et al., 2005) does not embrace all components of ACT (24/24 coverage being shared with outpatient emergency team).

Some EP trials (such as the Danish OPUS study (Bertelsen et al., 2008, 2009; Jeppesen et al., 2005; Nordentoft et al., 2015), or the English LEO (Craig et al., 2004; Tempier et al., 2012)) have shown that the implementation of assertive outreach alone improves some aspects of clinical and functional outcomes when compared to standard care. However, some other trials with similar settings have not found such benefits (Boden et al., 2010; Kuipers et al., 2004; Rosenbaum et al., 2005). This may be linked to differences in treatment strategies, considering that in some programs, ACT intervention is proposed to all EP patients and during the entire duration of treatment (Nordentoft et al., 2006) while in other programs, it is available only for a subgroup of patients (Brewer et al., 2015). A more selective prescription of ACT may be justified by the fact that EP patients are heterogeneous regarding the severity of their illness, 
their willingness to engage in treatments and their therapeutic needs. In addition, due to low case load per case managers and need for round the clock coverage, many programs can't afford to offer ACT to all patients. It remains however unclear which are the EP patients who need such an intervention, and which is their outcome when compared to patients treated with less intensive case management.

Some services, such as the Early Psychosis Prevention and Intervention (EPPIC (McGorry et al., 1996)) in Australia, or TIPP (Baumann et al., 2013) in Lausanne, Switzerland, provide case management for all patients, and ICM as an adjunct in a subgroup of them (Morandi and Bonsack, 2011). One recent study conducted at EPPIC (Brewer et al., 2015) examined the profile of EP patients who were offered ICM on the basis of the identification of a high-risk profile (poor engagement, low psychosocial support, persistent symptoms, high suicide risk, frequent relapses and risk-taking behaviors) in a real-world clinical setting. They compared 120 consecutive patients referred to ICM, with 50 patients receiving standard case management approach and found that ICM users were predominantly unemployed males with low levels of functioning and education. In addition, family history of mental illness, migration and exposure to trauma were highly prevalent in this subgroup. Data showed that ICM improved psychopathology and psychosocial outcomes and reduced risk taking, admissions, bed days and crisis contact. Despite these interesting findings, prospective evaluations were reported only within the ICM group, and the sample of patients treated with the standard CM approach was relatively small (50 patients); more data regarding such specific ICM intervention for selected EP patients is needed in order to better refine the profile of those who may benefit from such an approach.

With this in mind, and in the frame of a 3-years prospective naturalistic cohort of EP patients referred to TIPP (Baumann et al., 2013), we aimed to examine the prevalence, the characteristics, the clinical and functional outcomes, of a subgroup of "difficult to engage" 
patients who were offered additional ICM in the context of a specialized EP program. Our hypotheses were that: (1) A significant proportion of TIPP patients would need ICM intervention; (2) This subgroup of EP patients would have specific premorbid characteristics; (3) This subgroup would have a distinct clinical presentation at the time of entry to the program, and (4) The intervention process and outcome would be distinct compared to the other TIPP patients.

\section{Methods}

\subsection{Procedure and participants}

TIPP (Treatment and early Intervention in Psychosis Program), a specialized early psychosis program, was launched in 2004 at the Department of Psychiatry CHUV, in Lausanne, Switzerland (Baumann et al., 2013; Conus et al., 2008). Entry criteria to the program are: (I) aged between 18 and 35; (II) residing in the catchment area of about 300000 inhabitants; (III) meeting threshold criteria for psychosis, as defined by the 'Psychosis threshold' subscale of the Comprehensive Assessment of At Risk Mental States (CAARMS (Yung et al., 2005)) scale. Patients are referred to other treatment programs if they have been taking antipsychotic medication for more than a total of 6 months, have psychosis related to intoxication or organic brain disease, or have an intelligence quotient below 70. All patients are treated by a psychiatrist and a case manager, the latter being available for a maximum of 2 home visits per week. Every TIPP patient goes through a thorough clinical assessment at baseline and evaluations are conducted at regular interval both by the case manager and a clinical psychologist charged to evaluate symptoms on the basis of standard rating scales (see below) in order to objectively assess patient's evolution.

A subsample of TIPP patients receive additional support and treatment from the ICM team (called 'Soins Intensifs dans le milieu': SIM (Morandi and Bonsack, 2011)) at some point during the three years of TIPP (ICM patients), a period during which case managers nevertheless 
remain involved in order to warrant continuity of care (Baumann et al., 2013). ICM intervention in the frame of TIPP program is offered in three types of situations: (i) in case of need for assessment and engagement of patients who are treatment refractory or who need prolonged community assessment before they can be referred to the TIPP outpatient clinic, (ii) in case of need to provide time limited intensification of treatment when close monitoring is needed more than twice per week (such as daily monitoring of medication) and (iii) as an alternative to hospital admission when a relapse occurs (Baumann et al., 2013). The multidisciplinary ICM team is composed of 2.8 full-time equivalent (FTE) nurses, 1 FTE social worker and 1.8 FTE psychiatrists. The program's main characteristics are home-based interventions, a 1:10 caseload ratio, 5 weekly working days (with extendable hours as needed), individual caseloads with team backup, up to 2 daily contacts, assertive outreach, focus on engagement and systematic contact with relatives and other caregivers (Bonsack et al., 2005).

The Research and Ethics Committee of the Faculty of Biology and Medicine of Lausanne University granted access for research purposes to all data collected in the frame of clinical treatment. Therefore data stemming from the prospective clinical assessment of all patients treated at TIPP is accessible for research.

The current study is based on the prospective follow-up of the patients who entered TIPP between 2004 and January 2011; the sample was limited to this subgroup in order to include only patients who could have been followed-up for 36 months.

\subsection{Measures}

A specially designed questionnaire is completed for all patients enrolled in the program by case managers who have up to one hundred contacts with patients during the three years of treatment. It allows assessment of demographic characteristics, past medical history, exposure to life events as well as symptoms and functioning. It is completed on the basis of information 
gathered from patients and their family over the first few weeks of treatment and can be updated during follow up if new information emerges. Follow-up assessments exploring various aspects of treatment and co-morbidities as well as evolution of psychopathology and functional level are conducted by a research psychologist and by case managers at baseline, after 2, 6, 12, 18, 24, 30 and 36 months in treatment.

\subsection{Diagnostic Assessment}

Diagnosis is the result of an expert consensus and is based on the following elements: (1) Diagnosis reported by a treating psychiatrist in all medical documents and at the end of any hospitalization; (2) Longitudinal assessment by clinical case managers over the 3 years of treatment. The consensus diagnosis procedure is carried out by a senior psychiatrist and the senior psychologist who is in charge of scale based assessment over the treatment period. They both review the entire file once after 18 months and again after 36 months, or at the end of treatment. They then conduct a diagnostic process based on DSM-IV criteria (APA, 2000), discussing any unclear issue with the clinical case manager. In this paper, only the final diagnosis, defined at the end of the entire TIPP treatment period, is considered.

\subsection{Pre-treatment, baseline and outcome characteristics}

(i) Pre-treatment characteristics: The premorbid functioning was assessed using the Premorbid Adjustment Scale (PAS(Cannon-Spoor et al., 1982)). According to others (Alameda et al.), the data was used to come up with a global score as well as a subdivision between an academic and a social sub-score, both in the childhood and in early adolescence phases. Duration of untreated psychosis (DUP) was obtained on the basis of an expert consensus, based on information gathered with the patient and his relatives, and was defined as the time between the onset of psychotic symptoms and the date of entry in TIPP program (Polari et al., 2011). Past psychiatric diagnoses were assessed according to DSM-IV criteria, and past suicide attempts 
according to ICD-10 classification (Dilling and Dittmann, 1990). Past history of trauma was assessed by CMs on the basis of the knowledge of history of patients in the frame of a 3 year's trusting relationship, and based on interviews with relatives when indicated (Alameda et al.). Socio-economic status (SES) was subdivided into high, intermediate and low according to others (Chandola and Jenkinson, 2000). Lifetime diagnosis of substance abuse or dependence before the entering the program was rated by case managers based on the Diagnostic and Statistical Manual for Mental Disorders, Fourth Edition (APA, 2000). History of migration in adversity was considered when patients where migrants as political or economic refugee.

(ii) Patients characteristics at baseline: Patients were rated as "working" at entry (baseline) on the basis of the Modified Vocational Status Index (MVSI (Tohen et al., 2000): i.e., paid or unpaid full- or part-time employment, being an active student in school or university, or head of household with employed partner (homemaker), or full or part-time volunteer). Patients were rated as "living independently" on the basis of the Modified Location Code Index Independent living (MLCl (Tohen et al., 2000): i.e., head of household, living alone, with partner, or with peers, and living with family with minimal supervision). The level of functioning at baseline was estimated on the Global Assessment of Functioning (GAF(APA, 2000)) and with Social and Occupational Functioning Assessment Scale (SOFAS(APA, 2000)). While the SOFAS only takes the social and occupational functioning into account, the GAF also includes the intensity of symptoms. Severity of illness at baseline was assessed with the Clinical global impression scale (CGI(Guy, 1976)). Substance use at baseline was assessed in 2 ways: first, on the basis of DSM-IV criteria and second was dichotomized between "absent/light substance use" and "moderate to severe substance use" on the basis of the case manager rating Scale (CMRS(Drake et al., 1990)) that ranges from 1 to 5,1 being absence of substance use and 5 very severe substance use. Insight into illness was assessed on the basis of a three points scale of ranging from absent, to partial and complete (Conus et al., 2007). 
(iii) Outcome measures after 2, 6, 12, 18, 24, 30 and 36 months of follow-up: The functional level was assessed with the GAF and SOFAS scales. Degree of insight and level of substance abuse were assessed at each time point on the basis of the scale described above. Treatment adherence was estimated by case managers and treating psychiatrist in a consensus approach and scored on the basis of a 3 point Treatment Adherence Scale (TAS) ranging from 0 to $2(0=$ non adherence; 1 = partial adherence (from $25 \%$ to $75 \%$ of the time during the evaluation period); and $2=$ total adherence (from $75 \%$ to $100 \%$ of the time during the evaluation period). It was estimated after two months of treatment and at each time point until the end of the followup. Intensity of illness was assessed on the basis of the CGI at each time point. Additionally the following psychopathological measures were assessed at each time point: 1) the level of positive and negative symptoms, using the total score of the positive and negative components of the Positive and Negative Symptoms Scale (PANSS(Kay et al., 1987)); 2) the level of depressive symptoms, using the total score of the Montgomery-Asberg Depression Rating Scale (MADRS(Montgomery and Asberg, 1979)); 3) the level of manic symptoms, using the total score of the Young Mania rating Scale, (YMRS(Young et al., 1978)).

\subsection{Outcomes definitions at discharge}

Symptomatic remission at discharge was based on the Andreasen Criteria (Andreasen et al., 2005): the simultaneous ratings of mild or less $(\leq 3)$ on selected PANSS' items (delusion, unusual thought content, hallucinatory behavior, conceptual disorganization, mannerisms, blunted affect, social withdrawal and lack of spontaneity) maintained over 6 months. Functional recovery was operationalized as a final PAS score inferior or equal to the premorbid rating on four of the five items of the PAS general scale. Ratings on education and abruptness in the change in work were not included in this definition, as they could not have changed during the outcome period, in keeping others (Conus et al., 2006; Strakowski et al., 1998). Disengagement 
rates were calculated at discharge and were defined as a patient's refusal to meet with clinicians, despite multiple attempts on their part to engage the patient in treatment .

\subsection{Statistical analysis}

Comparisons in terms of pre-treatment, baseline, follow-up and at discharge characteristics between the ACT and the standard CM subsamples were performed with independent t-tests for continuous variables and Pearson's Chi-Square tests (or Fisher Exact tests when appropriate) for categorical variables. Because DUP values were highly skewed, comparisons were performed using Mann-Whitney U-tests. Data are presented descriptively using mean (M) and standard deviation (SD) or median (Mdn) and interquartile range (IQR) for the DUP values. All statistical analyses were performed with IBM SPSS version 22. All statistical tests were twotailed and significance was determined at the .05 level.

\section{Results}

The results are organized as follows: A) pretreatment and baseline characteristics, B) clinical, functional and substance use outcomes during follow-up and C) outcomes at discharge.

\subsection{Patients sample, timing of SIM intervention, pretreatment and baseline factors.}

Of the first 229 patients included in the study, 60 (26.2\%) were followed by SIM at some point of the follow up (ICM-patients); 169 (73.8\%) were followed with standard TIPP case management exclusively (TIPP-patients). $75 \%$ of ICM interventions started in the first three months of treatment; Their duration was short in $30.0 \%$ ( 0 to 6 months), intermediate in $40.0 \%$ (6 months to 2 years) and long in 30\%(2 to 3 years). Comparisons between ICM- and TIPP-patients regarding pretreatment and baseline characteristics are reported in Table 1. Compared to TIPPpatients, ICM-patients showed a poorer level of premorbid functioning on the PAS Total score 
$(p=0.028)$, the PAS Early Adolescence score $(p=0.010)$ the PAS Early Adolescence Academic score $(p=0.019)$ and the Academic total score $(p=0.043)$. ICM-patients were more likely to have a lifetime diagnosis of dependence to alcohol $(p=0.043)$ and to cannabis $(p=0.040)$ and to have a history of migration in adversity $(p=0.033)$.

\subsection{Clinical, functional, substance use outcomes during the follow-up.}

The comparison in terms of MVSI and MLCI status, CGI, insight, MADRS, YMRS and CMRS (for alcohol, cannabis and substances) levels at baseline, at 18 months (except for CGI) and at discharge between TIPP and ICM-patients are presented in table 2. The complete trajectories in terms of GAF, SOFAS, PANSS positive and PANSS negative are also illustrated in figure 1. Comparisons between TIPP-patients and ICM-patients during the follow-up revealed that ICMpatients: 1) Were less often working at entry in TIPP, which was globally maintained during the follow-up ( $p=0.001$ at entry, $p=0.060$ at 18 months and $p=0.032$ at discharge); 2 ) Were less often living independently at entry at trend level $(p=0.059)$ which disappeared during the follow up ( $p=0.467$ at discharge) 3) Had the same low level of insight at baseline $(p=0.076)$, which temporarily differed during the follow-up (lesser degree of insight for ICM-patients at 18 month, $p=0.009)$ but became again not significantly different at discharge $(p=0.067)$; 4) Used more alcohol and cannabis at baseline $(p=0.022$ for alcohol and $p=0.003$ for cannabis) but not consistently during the follow-up (non significant at 18 months for both substances and significant only for cannabis at discharge $p=0.029)$ 5) Did not differ in terms of substance use at baseline and follow-up. Nevertheless, ICM-patients significantly used more substances at discharge $(p=0.023) ; 6)$ Showed a higher severity of illness as measured with the CGI as baseline $(p \leq 0.001)$ and at discharge $(<0.012)$; 7$)$ Did not show more positive or negative symptoms as measured with the PANSS in the beginning of the follow-up. However, as illustrated in figure 1, differences emerged only in the second part of the follow up and then remained significant until discharge $(p=0.026$ for PANSS positive; $p=0.017$ for PANSS 
negative); 7) Had significantly poorer levels of functioning as measured with SOFAS and GAF at any time (as illustrated in figure 1);

\subsection{Functional and clinical outcomes at discharge.}

TIPP-patients and ICM-patients differed in terms of symptomatic remission at discharge according to Andreasen's criteria: $56.4 \%$ of TIPP-patients were symptomatically remitted in comparison with only $27.9 \%$ of ICM-patients $(p=0.001)$. Patients did not differ in terms of functional recovery at discharge $(p=0.364)$ on the basis of a return to pre-morbid functional level on the PAS (Conus et al., 2006).

\subsection{Number of hospitalizations and treatment adherence during the follow-up}

As shown in Figure 2, after two months of treatment $43.9 \%$ of ICM-patients had a complete adherence to treatment while this was the case for $65.2 \%$ of TIPP-only patients $(p=0.005)$. This difference remained significant during 6 months of treatment and disappeared after this time point. At the end of the follow-up, $91.9 \%$ of TIPP-patients and $89.0 \%$ of ICM-patients $(p=0.652)$ were adherent to treatment. In order to allow comparisons with previous studies, we calculated the disengagement rates at discharge, which was of $13.9 \%$ for the overall sample, $12.8 \%$ in TIPP group, and $16.3 \%$ in ICM-group ( $p=0.551)$. TIPP-

TIPP- patients were more likely to be entered in TIPP program through an initial admission to hospital, while ICM-patients were more often hospitalized for the first time only later during treatment.

\section{Discussion}

The purpose of this study was to examine the prevalence, the characteristics, the clinical and the functional outcome, of a subgroup of "difficult to engage" patients who were offered additional ICM in the context of a specialized 3-years EP program. In line with our first 
hypothesis, we found that a substantial subgroup of patients treated at TIPP (60 of the 229; $26.2 \%)$ had been offered addition of ICM to standard case management. This rate is higher than that reported by Brewer et al.(Brewer et al., 2015) at EPPIC who found that only $9.8 \%$ of patients were accepted by their intensive case management team. Obviously, the identification process applied at TIPP to identify patients referred to ICM is not standardized and indications are therefore dependent on various factors among which the case load of the ICM team at the moment of referral. However, the fact that, as discussed below, these patients have a specific profile compared to those who were not offered ICM shows that a population with specific needs for more intensive care can be identified. It also suggests that an important proportion of EP patient may not need such intensive care, considering they do significantly better than ICMpatients despite absence of intensive assertive outreach; this is important to know where costs need to be considered when developing a specialized EP program. Studies conducted in a different setting with a randomized design are however needed to provide better ways to identify EP patients who need ICM intervention.

In keeping with our second hypothesis, we found that ICM-patients had specific premorbid characteristics when compared to the other EP patients. ICM-patients had a poorer premorbid academic functional level during the early adolescence, higher rates of unemployment, were more likely to have a history of adverse migration and of alcohol and cannabis use before the onset of psychosis. These results are in line with a previous publication (Brewer et al., 2015) where authors found that EP patients needing an intensive approach had lower functioning, and more likely to be unemployed and to have faced migration. However, contrary to the EPPIC study, ICM-patients at TIPP did not differ in terms of rates of both family history of mental illness and exposure to trauma. This may be linked to various factors such as sample size or social context where the study was conducted: whatever the case may be, more studies are needed to determine if these elements should to be considered when trying to identify EP patients in need 
of intensive care. In addition, according to others (Alameda et al.), we explored premorbid functioning in more detail, distinguishing academic and social dimensions, and found that if ICM-patients had similar global social premorbid level of functioning compared to general EP patients, they perform significantly less well in the academic domain during early adolescence but that these differences were not present in childhood. This may be explained by the additional observation that ICM-patients were more likely to have used alcohol and cannabis before illness onset, considering adolescence is a vulnerable time for the adverse mental effects of cannabis(Johns, 2001), which was shown to increase the risk for developing psychosis (Arseneault et al., 2002), especially when used at an early age (Stefanis et al., 2004). Furthermore, previous studies in EP have shown that cannabis use correlates with poorer academic, but not social functioning in early adolescence (Compton et al., 2011). Even though we cannot infer causality, our results are in line with this evidence and suggest that early substance use and its impact on functional level may be an element driving the need for intensification of treatment after psychosis onset.

Confirming our third hypothesis, ICM-patients had a different baseline clinical profile when compared to the other EP patients. When entering the TIPP program, they had lower level of insight, higher intensity of illness on the basis of CGI scores, lower levels of functioning and they were more likely to use alcohol and cannabis. This suggests that clinical profile at baseline may also contribute to the identification of this subgroup in order to refine the indication to ICM. It also illustrates that clinicians dealing with such patients in ICM context need to face an important number of challenges, which justifies the low case load generally applied in such teams.

Our fourth aim was to explore outcome differences between both groups. We observed that ICM-patients were less likely to be adherent to medication during the early phase of TIPP treatment; however, differences disappeared during follow-up suggesting that SIM intervention 
contributed to this improvement. After 12 months in TIPP, around $75 \%$ of patients were engaged into antipsychotic treatment and at discharge $91.9 \%$ of TIPP patients and $89 \%$ of ICM patients had a complete adherence to antipsychotics. At discharge, only $2.3 \%$ of patients in the overall sample could never be approached by the members of TIPP and the overall disengagement rates at discharge were similar between groups (13.9\% in the overall sample). Previous programs specialized in early intervention in psychosis have reported disengagements rates for shorter observation periods, ranging between $18 \%$ and $25 \%$ for a 12 months observation period (Craig et al., 2004; Malla and Norman, 2001; Turner et al., 2007), and 23\% for a 18 months observation period (Conus et al., 2010). Our results over a 36-month period shows that standard and intensive case management are efficient tools to promote engagement in the early phase of psychotic disorders, event in patients with very complex clinical and social issues. Despite this, ICM-patients unfortunately maintained higher levels of positive and negative symptoms, a lower level of functioning and a higher rate of substance use until the end of the program when compared to the other EP patients treated with regular CM approach. ICMpatients may belong to a subgroup of patients suffering from a more severe form of disorder with less potential for full recovery. It is well established that despite good rate of engagement in treatment, the persistence of substance abuse certainly plays a major role in limitation of recovery (Lambert et al., 2005) In addition, it is possible that the compound effect of delayed engagement in treatment and DUP lead to the fact that exposure to treatment unfortunately occurs well after the closure of a potential critical phase where outcome can be significantly influenced. However, the good rate of engagement even in the context of very complex situation gives a signal that exposure to treatment is also possible for these patients and that an effort for an earlier intervention should be made. Finally, the important deficit in functional level in this subgroup may also explain part of this poorer outcome. New strategies should be implemented to facilitate social and vocational integration of such patients, and supported employment seems to be promising in this regard. 
This study has various limitations. Firstly, treatment adherence was based on case manager's clinical judgment. Such indirect methods are the most frequently used in clinical trials, but it is well established that they may overestimate level of compliance. Ideally, studies should assess medication adherence through multiple methods including plasmatic dosage or electronic pill counts. However, many patients, especially those needing ICM, would not have sufficient degree of collaboration use these devices, and a restriction to patients with sufficient collaboration would induce a very important bias in the study. Secondly, the study being a naturalistic follow-up of patients treated in real-world treatment, the number of patients who were assessed for psychopathology during the follow-up varies between time points, which should be considered in the interpretation of our figures.

Despite these limitations, our data suggests that in a sample of EP patients treated in a specialized program, clinicians can identify a subgroup of patients who need intensification of case management. Our data suggest that difficult to engage patients can reach good levels of engagement and adherence to treatment. However, their poorer symptomatic and functional outcome suggests the need for adapted intervention, especially regarding substance use disorder. Finally, our data suggests as well that if ICM is a very useful tool for this subgroup of patients, the majority of EP patients may not need such an intensive intervention and may do well regarding engagement and adherence to treatment with less intensive follow-up. This may be an important element to keep in mind when costs become an obstacle when negotiating funding in order to implement early intervention programs.

\section{Role of funding source}


Swiss National Science Foundation (\#320030_122419 to P.C. and KQD); National Center of Competence in Research (NCCR) "SYNAPSY — The Synaptic Bases of Mental Diseases" from the Swiss National Science Foundation (no. 51AU40_125759 to KQD); Avina Foundation.

\section{Acknowledgments}

We thank to the Case Managers from Tipp Program for collecting this data over the years. Most of all, we express our gratitude to all patients for their enduring participation. We thank our financial supports. The authors have declared that there are no conflicts of interest in relation to the subject of the study.

Alameda, L., Ferrari, C., Baumann, P., Gholam-Rezaee, M., Do, K., Conus, P., Childhood sexual and physical abuse: age at exposure modulates impact on functional outcome in early psychosis patients. Psychol Med, 1-10.

Andreasen, N.C., Carpenter, W.T., Jr., Kane, J.M., Lasser, R.A., Marder, S.R., Weinberger, D.R., 2005. Remission in schizophrenia: proposed criteria and rationale for consensus. Am J Psychiatry 162 (3), 441-449.

APA, 2000. DSMIV-TR. Washington, DC : American Psychiatric Association, 2000.

Arseneault, L., Cannon, M., Poulton, R., Murray, R., Caspi, A., Moffitt, T.E., 2002. Cannabis use in adolescence and risk for adult psychosis: longitudinal prospective study. BMJ 325 (7374), 12121213.

Baumann, P.S., Crespi, S., Marion-Veyron, R., Solida, A., Thonney, J., Favrod, J., Bonsack, C., Do, K.Q., Conus, P., 2013. Treatment and Early Intervention in Psychosis Program (TIPP-Lausanne): implementation of an early intervention programme for psychosis in Switzerland. Early Interv Psychiatry.

Bertelsen, M., Jeppesen, P., Petersen, L., Thorup, A., Ohlenschlaeger, J., le Quach, P., Christensen, T.O., Krarup, G., Jorgensen, P., Nordentoft, M., 2008. Five-year follow-up of a randomized multicenter trial of intensive early intervention vs standard treatment for patients with a first episode of psychotic illness: the OPUS trial. Arch Gen Psychiatry 65 (7), 762-771.

Bertelsen, M., Jeppesen, P., Petersen, L., Thorup, A., Ohlenschlaeger, J., le Quach, P., Christensen, T.O., Krarup, G., Jorgensen, P., Nordentoft, M., 2009. [First episode of psychosis intensive early intervention programme versus standard treatment--secondary publication]. Ugeskr Laeger 171 (41), 2992-2995.

Boden, R., Sundstrom, J., Lindstrom, E., Wieselgren, I.M., Lindstrom, L., 2010. Five-year outcome of firstepisode psychosis before and after the implementation of a modified assertive community treatment programme. Soc Psychiatry Psychiatr Epidemiol 45 (6), 665-674. 
Bonsack, C., Adam, L., Haefliger, T., Besson, J., Conus, P., 2005. Difficult-to-engage patients: a specific target for time-limited assertive outreach in a Swiss setting. Can J Psychiatry 50 (13), 845-850.

Brewer, W.J., Lambert, T.J., Witt, K., Dileo, J., Duff, C., Crlenjak, C., McGorry, P.D., Murphy, B.P., 2015. Intensive case management for high-risk patients with first-episode psychosis: service model and outcomes. The Lancet Psychiatry 2 (1), 29-37.

Cannon-Spoor, H.E., Potkin, S.G., Wyatt, R.J., 1982. Measurement of premorbid adjustment in chronic schizophrenia. Schizophr Bull 8 (3), 470-484.

Chandola, T., Jenkinson, C., 2000. The new UK National Statistics Socio-Economic Classification (NS-SEC); investigating social class differences in self-reported health status. J Public Health Med 22 (2), 182-190.

Compton, M.T., Broussard, B., Ramsay, C.E., Stewart, T., 2011. Pre-illness cannabis use and the early course of nonaffective psychotic disorders: associations with premorbid functioning, the prodrome, and mode of onset of psychosis. Schizophr Res 126 (1), 71-76.

Conus, P., Cotton, S., Abdel-Baki, A., Lambert, M., Berk, M., McGorry, P.D., 2006. Symptomatic and functional outcome 12 months after a first episode of psychotic mania: barriers to recovery in a catchment area sample. Bipolar Disord 8 (3), 221-231.

Conus, P., Cotton, S., Schimmelmann, B.G., McGorry, P.D., Lambert, M., 2007. The First-Episode Psychosis Outcome Study: premorbid and baseline characteristics of an epidemiological cohort of 661 first-episode psychosis patients. Early Interv Psychiatry 1 (2), 191-200.

Conus, P., Lambert, M., Cotton, S., Bonsack, C., McGorry, P.D., Schimmelmann, B.G., 2010. Rate and predictors of service disengagement in an epidemiological first-episode psychosis cohort. Schizophr Res 118 (1), 256-263.

Conus, P., Montagrin, Y., Bircher, R., Sarrasin, P., Polari, A., Bonsack, C., 2008. 118-TIPP-Lausanne first episode psychosis program: Patients' baseline characteristics and impact of the program on adherence to psychosocial treatment. Schizophr Res 98, 81-82.

Craig, T.K., Garety, P., Power, P., Rahaman, N., Colbert, S., Fornells-Ambrojo, M., Dunn, G., 2004. The Lambeth Early Onset (LEO) Team: randomised controlled trial of the effectiveness of specialised care for early psychosis. BMJ 329 (7474), 1067.

Dilling, H., Dittmann, V., 1990. [Psychiatric diagnosis following the 10th revision of the International Classification of Diseases (ICD-10)]. Nervenarzt 61 (5), 259-270.

Drake, R.E., Osher, F.C., Noordsy, D.L., Hurlbut, S.C., Teague, G.B., Beaudett, M.S., 1990. Diagnosis of alcohol use disorders in schizophrenia. Schizophr Bull 16 (1), 57-67.

Guy, W., 1976. ECDEU assessment manual for psychopharmacology. US Department of Health, Education, and Welfare, Public Health Service, Alcohol, Drug Abuse, and Mental Health Administration, National Institute of Mental Health, Psychopharmacology Research Branch, Division of Extramural Research Programs.

Jeppesen, P., Petersen, L., Thorup, A., Abel, M.B., Oehlenschlaeger, J., Christensen, T.O., Krarup, G., Hemmingsen, R., Jorgensen, P., Nordentoft, M., 2005. Integrated treatment of first-episode psychosis: effect of treatment on family burden: OPUS trial. Br J Psychiatry Suppl 48, s85-90.

Johns, A., 2001. Psychiatric effects of cannabis. The British Journal of Psychiatry 178 (2), 116-122.

Kay, S.R., Fiszbein, A., Opler, L.A., 1987. The positive and negative syndrome scale (PANSS) for schizophrenia. Schizophr Bull 13 (2), 261-276.

Kuipers, E., Holloway, F., Rabe-Hesketh, S., Tennakoon, L., Croydon, O., Assertive Support, T., 2004. An RCT of early intervention in psychosis: Croydon Outreach and Assertive Support Team (COAST). Soc Psychiatry Psychiatr Epidemiol 39 (5), 358-363.

Lamb, H.R., 1980. Therapist-case managers: more than brokers of services. Psychiatric Services 31 (11), 762-764. 
Lambert, M., Conus, P., Lubman, D., Wade, D., Yuen, H., Moritz, S., Naber, D., McGorry, P., Schimmelmann, B., 2005. The impact of substance use disorders on clinical outcome in 643 patients with first-episode psychosis. Acta Psychiatr Scand 112 (2), 141-148.

Malla, A.M., Norman, R., 2001. Treating psychosis: is there more to early intervention than intervening early? Canadian Journal of Psychiatry 46 (7), 645-648.

Marion-Veyron, R., Mebdouhi, N., Baumann, P.S., Thonney, J., Crespi, S., Conus, P., 2013. Les premiers épisodes psychotiques: de l'importance du case management. L'Évolution Psychiatrique 78 (1), 41-51.

Marshall, M., Lockwood, A., 1998. Assertive community treatment for people with severe mental disorders. Cochrane database of systematic reviews 2, --•.

Marshall, M., Lockwood, A., Lewis, S., Fiander, M., 2004. Essential elements of an early intervention service for psychosis: the opinions of expert clinicians. BMC Psychiatry 4, 17.

Marshall, M., Rathbone, J., 2011. Early intervention for psychosis. Cochrane Database Syst Rev(6), CD004718.

McGorry, P.D., Edwards, J., Mihalopoulos, C., Harrigan, S.M., Jackson, H.J., 1996. EPPIC: an evolving system of early detection and optimal management. Schizophr Bull 22 (2), 305-326.

Montgomery, S.A., Asberg, M., 1979. A new depression scale designed to be sensitive to change. $\mathrm{Br}$ J Psychiatry 134, 382-389.

Morandi, S., Bonsack, C., 2011. Engager dans les soins des personnes souffrant de schizophrénie par un suivi intensif dans le milieu (SIM). SCHWEIZERARCHIVF Ü RNEUROLOGIEUNDPSYCHIA TRIE 1 (6), 2.

Mueser, K.T., Bond, G.R., Drake, R.E., Resnick, S.G., 1998. Models of community care for severe mental illness: a review of research on case management. Schizophr Bull 24 (1), 37-74.

Nordentoft, M., 2015. Specialised assertive intervention in early psychosis. The Lancet Psychiatry 2 (1), 2-3.

Nordentoft, M., Melau, M., Iversen, T., Petersen, L., Jeppesen, P., Thorup, A., Bertelsen, M., Hjorthøj, C.R., Hastrup, L.H., Jørgensen, P., 2015. From research to practice: how OPUS treatment was accepted and implemented throughout Denmark. Early Interv Psychiatry 9 (2), 156-162.

Nordentoft, M., Petersen, L., Jeppesen, P., Thorup, A.A., Abel, M.B., Ohlenschlaeger, J., Christensen, T.O., Krarup, G., Jorgensen, P., 2006. [OPUS: a randomised multicenter trial of integrated versus standard treatment for patients with a first-episode psychosis--secondary publication]. Ugeskr Laeger 168 (4), 381-384.

Pepper, B., Kirshner, M.C., Ryglewicz, H., 2014. The young adult chronic patient: Overview of a population. Psychiatric Services.

Polari, A., Lavoie, S., Sarrasin, P., Pellanda, V., Cotton, S., Conus, P., 2011. Duration of untreated psychosis: a proposition regarding treatment definition. Early Interv Psychiatry 5 (4), 301-308.

Rosenbaum, B., Valbak, K., Harder, S., Knudsen, P., Koster, A., Lajer, M., Lindhardt, A., Winther, G., Petersen, L., Jorgensen, P., Nordentoft, M., Andreasen, A.H., 2005. The Danish National Schizophrenia Project: prospective, comparative longitudinal treatment study of first-episode psychosis. Br J Psychiatry 186, 394-399.

Stefanis, N., Delespaul, P., Henquet, C., Bakoula, C., Stefanis, C., Van Os, J., 2004. Early adolescent cannabis exposure and positive and negative dimensions of psychosis. Addiction 99 (10), 13331341.

Strakowski, S.M., Keck, P.E., McElroy, S.L., West, S.A., Sax, K.W., Hawkins, J.M., Kmetz, G.F., Upadhyaya, V.H., Tugrul, K.C., Bourne, M.L., 1998. Twelve-month outcome after a first hospitalization for affective psychosis. Arch Gen Psychiatry 55 (1), 49-55. 
Tempier, R., Balbuena, L., Garety, P., Craig, T.J., 2012. Does assertive community outreach improve social support? Results from the Lambeth Study of early-episode psychosis. Psychiatr Serv 63 (3), 216-222.

Tohen, M., Hennen, J., Zarate, C.M., Jr., Baldessarini, R.J., Strakowski, S.M., Stoll, A.L., Faedda, G.L., Suppes, T., Gebre-Medhin, P., Cohen, B.M., 2000. Two-year syndromal and functional recovery in 219 cases of first-episode major affective disorder with psychotic features. Am J Psychiatry 157 (2), 220-228.

Turner, M., Smith-Hamel, C., Mulder, R., 2007. Prediction of twelve-month service disengagement from an early intervention in psychosis service. Early Interv Psychiatry 1 (3), 276-281.

Veldhuizen, J.R., 2007. FACT: A Dutch Version of ACT. Community Mental Health Journal 43 (4), 421-433.

Young, R.C., Biggs, J.T., Ziegler, V.E., Meyer, D.A., 1978. A rating scale for mania: reliability, validity and sensitivity. Br J Psychiatry 133, 429-435.

Yung, A.R., Yuen, H.P., McGorry, P.D., Phillips, L.J., Kelly, D., Dell'Olio, M., Francey, S.M., Cosgrave, E.M., Killackey, E., Stanford, C., Godfrey, K., Buckby, J., 2005. Mapping the onset of psychosis: the Comprehensive Assessment of At-Risk Mental States. Aust N Z J Psychiatry 39 (11-12), 964-971.

Ziguras, S.J., Stuart, G.W., 2000. A meta-analysis of the effectiveness of mental health case management over 20 years. Psychiatric Services 51 (11), 1410-1421. 
Figure 1. Comparisons between TIPP and TIPP + SIM patients for PANSS positive and negative scores, GAF and SOFAS during the follow-up.
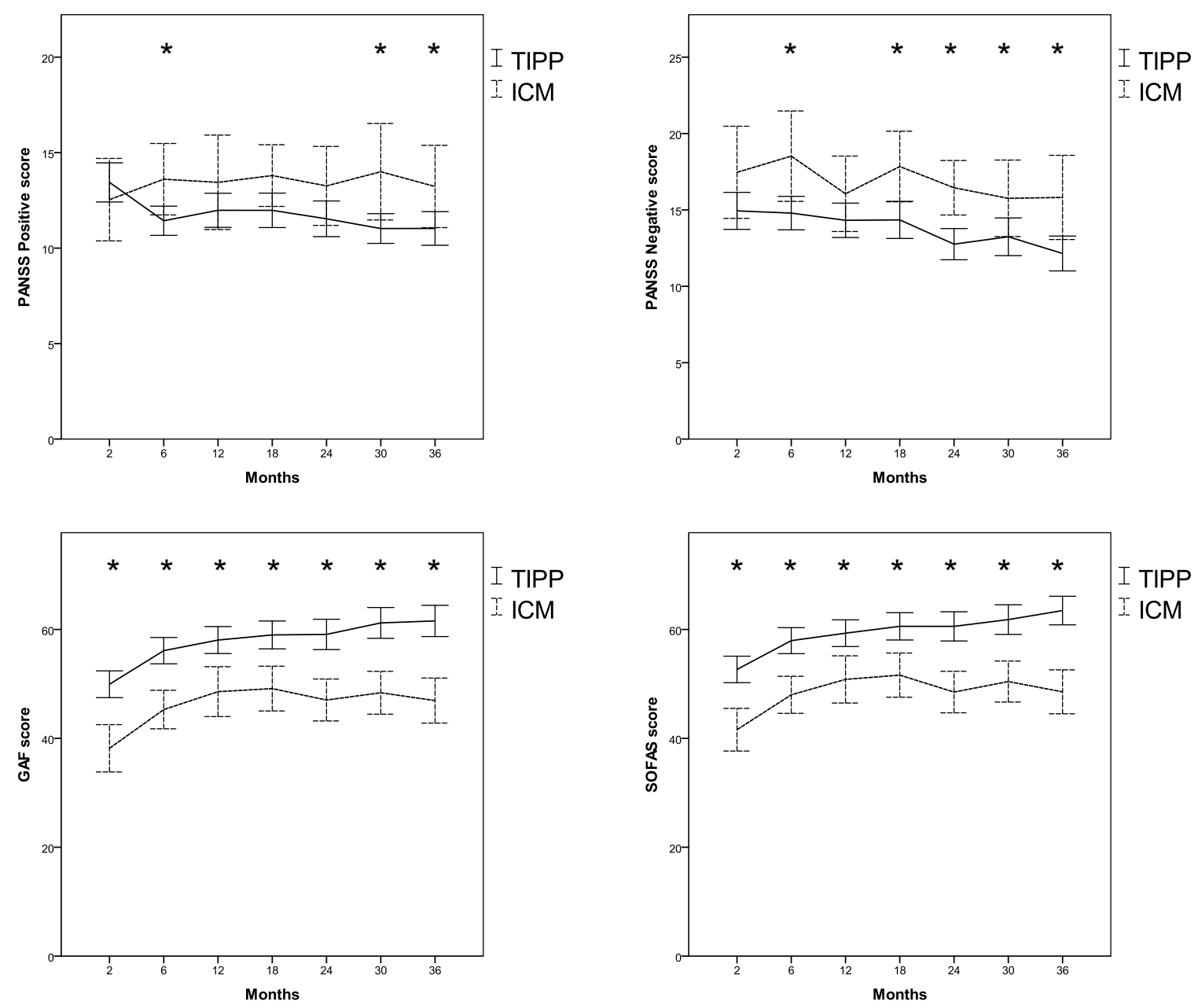

Note. ${ }^{*} p<$.05. PANSS: Positive and Negative Syndrome Scale. GAF: Global Assessment of Functioning Scale. SOFAS: Social and Occupational Assessment of functioning Scale. 
Figure 2. Comparisons between TIPP and ICM patients for complete adherence to antipsychotics during the follow-up.




Table 1. Pre-treatment and baseline characteristics of TIPP patients compared to ICM patients.

\begin{tabular}{|c|c|c|c|c|c|}
\hline & \multirow{2}{*}{$\begin{array}{c}\text { Total } \\
\mathrm{N}=229 \\
(100 \%)\end{array}$} & \multirow{2}{*}{$\begin{array}{c}\text { TIPP } \\
\\
N=169 \\
(73.8 \%)\end{array}$} & \multirow{2}{*}{$\begin{array}{c}\mathrm{ICM} \\
\mathrm{N}=60(26.2 \%)\end{array}$} & \multicolumn{2}{|c|}{ TIPP vs ICM } \\
\hline & & & & Stat. & P-value \\
\hline Age in year, $M(S D)$ & $24.10(4.88)$ & $24.19(4.88)$ & $23.83(4.90)$ & $\mathrm{t}(227)=0.485$ & .628 \\
\hline Sex,\%male $(N)$ & $157(68.6)$ & $66.9(113)$ & $73.3(44)$ & $\chi^{2}(1)=0.860$ & .354 \\
\hline \multicolumn{6}{|l|}{ SES, \% (N) } \\
\hline Low & $16.6(38)$ & $15.4(26)$ & $20.0(12)$ & $\chi^{2}(2)=0.723$ & .697 \\
\hline Intermediate. & $45.9(105)$ & $46.2(78)$ & $45.0(27)$ & & \\
\hline High & $37.6(86)$ & $38.5(65)$ & $35.0(21)$ & & \\
\hline \multicolumn{6}{|l|}{ Trauma, \% (N) } \\
\hline (non exposed) & $64.6(148)$ & $63.3(107)$ & $68.3(41)$ & $\chi^{2}(1)=0.488$ & .485 \\
\hline \multicolumn{6}{|l|}{ PAS scores, M (SD) } \\
\hline Childhood & $0.31(0.19)$ & $0.29(0.19)$ & $0.35(0.19)$ & $t(183)=-1.716$ & .088 \\
\hline Childhood Social & $0.30(0.24)$ & $0.29(0.24)$ & $0.34(0.26)$ & $t(194)=-1.259$ & .210 \\
\hline Childhood Academic & $0.33(0.22)$ & $0.32(0.23)$ & $0.36(0.19)$ & $t(189)=-1.162$ & .247 \\
\hline Early adolescence & $0.33(0.18)$ & $0.31(0.17)$ & $0.38(0.18)$ & $t(184)=-2.599$ & .010 \\
\hline Early adolescence Social & $0.29(0.22)$ & $0.27(0.22)$ & $0.34(0.21)$ & $t(192)=-1.727$ & .086 \\
\hline Early adolescence Academic & $0.39(0.23)$ & $0.37(0.22)$ & $0.46(0.23)$ & $t(192)=-2.366$ & .019 \\
\hline Social & $0.29(0.21)$ & $0.28(0.21)$ & $0.34(0.23)$ & $t(184)=-1.637$ & .103 \\
\hline Academic & $0.36(0.20)$ & $0.34(0.20)$ & $0.41(0.19)$ & $t(183)=-2.037$ & .043 \\
\hline Total & $0.32(0.17)$ & $0.30(0.17)$ & $0.37(0.18)$ & $t(172)=-2.212$ & .028 \\
\hline \multicolumn{6}{|l|}{$\begin{array}{l}\text { Lifetime substance } \\
\text { dependence, \% (N) }\end{array}$} \\
\hline \multirow{2}{*}{ Alcohol } & $9.3(20)$ & $6.9(11)$ & $16.1(9)$ & & .043 \\
\hline & $31.3(67)$ & $27.4(43)$ & $42.1(24)$ & $\chi^{2}(1)=4.112$ & .040 \\
\hline
\end{tabular}


Cannabis

Other Substances ${ }^{a}$

Diagnostic, \% (N)

Schizophrenia

Schizophreniform/BPE

Schizo-affectif disorder

Major depression ${ }^{\mathrm{c}}$

Bipolar disorder

Others

Past psychiatric history, \% (N)

$67.3(152)$

$65.7(109)$

71.7 (43)

$\chi^{2}(1)=0.721$

.396

Past forensic history, \% (N)
$1.7(4)$

127.5

(523.25)

$53.8(121)$

$57.0(94)$

$27.9(29)$

109.0

(631.50)

$33.1(48)$
$10.2(6)$

$\chi^{2}(1)=4.211$

.397

$\chi^{2}(1)=0.717$
71.7 (43)

$5.0(3)$

$13.3(8)$

$1.7(1)$

$3.3(2)$

$5.0(3)$

b

.170<smiles>CCC</smiles>

DUP, Mdn (IQR)

205.5 (649.25)

$U=4643.0$

Migration (Born in $\mathrm{CH}$ )

46.3 (19)

$\chi^{2}(1)=4.524$

.033

Note. $a=$ non prescribed drugs and narcotics. $b=$ Fisher Exact Test. PAS: Premorbid Adjustment Scale. DUP: Duration of Untreated Psychosis. BPE: Brief psychotic episode. $c=$ with psychotic features. SES: socio-economic status. IQR = Interquartile range. $\mathrm{CH}$ : Switzerland. 
Table 2 Comparisons of clinical, psychosocial, substance use outcomes and engagement into care at baseline, after 18 month and at discharge (36 month) between TIPP patients with ICM patients.

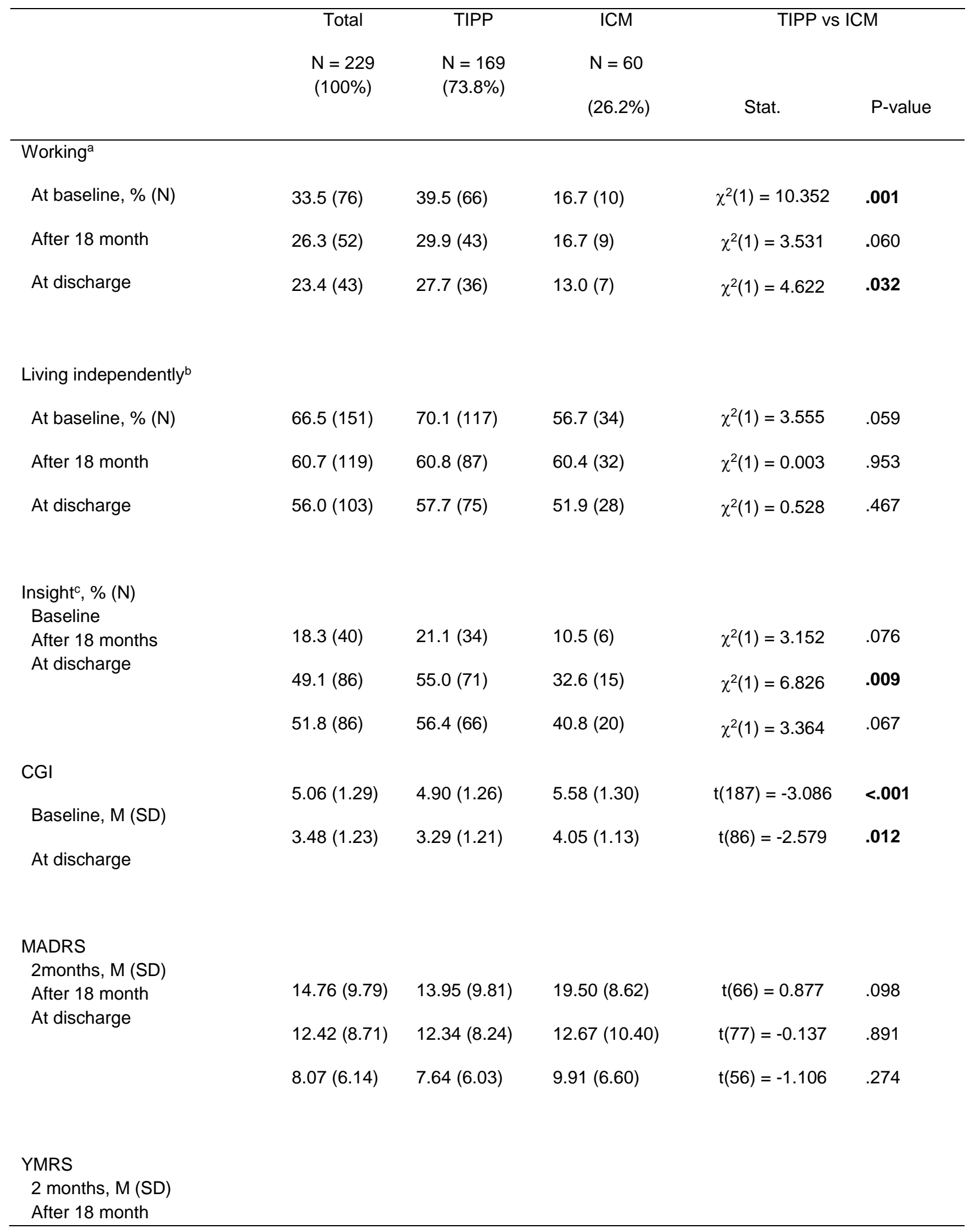




\begin{tabular}{|c|c|c|c|c|c|}
\hline \multirow[t]{3}{*}{ At discharge } & $6.10(5.68)$ & $6.03(5.49)$ & $6.54(7.10)$ & $t(101)=-0.298$ & .766 \\
\hline & $5.81(5.36)$ & $5.55(4.87)$ & $6.76(6.85)$ & $t(31.2)=-0.830$ & .413 \\
\hline & $5.74(5.27)$ & $5.68(5.01)$ & $5.91(6.12)$ & $t(86)=-0.174$ & .862 \\
\hline \multicolumn{6}{|l|}{$\mathrm{CMRS}^{\mathrm{d}}$ Baseline, \% (N) } \\
\hline Alcool & $14.4(33)$ & $11.2(19)$ & $23.3(14)$ & $\chi^{2}(1)=5.248$ & .022 \\
\hline Cannabis & $24.0(55)$ & $18.9(32)$ & $38.3(23)$ & $\chi^{2}(1)=9.130$ & .003 \\
\hline Substances $^{e}$ & $6.6(15)$ & $5.9(19)$ & $8.3(5)$ & $f$ & .547 \\
\hline \multicolumn{6}{|l|}{$\mathrm{CMRS}^{\mathrm{d}}$ after 18 month } \\
\hline Alcool & $5.5(11)$ & $3.4(5)$ & $11.1(6)$ & $f$ & .073 \\
\hline Cannabis & $10.1(20)$ & $7.6(11)$ & $16.7(9)$ & $\chi^{2}(1)=3.598$ & .058 \\
\hline Substances ${ }^{\mathrm{e}}$ & $4.5(0)$ & $2.8(4)$ & $9.5(5)$ & $f$ & .063 \\
\hline \multicolumn{6}{|l|}{$\mathrm{CMRS}^{\mathrm{d}}$ at discharge } \\
\hline Alcool & $6.6(12)$ & $4.5(6)$ & $11.1(6)$ & $f$ & .110 \\
\hline Cannabis & $10.8(20)$ & $7.6(10)$ & $18.5(10)$ & $\chi^{2}(1)=4.782$ & .029 \\
\hline Substances ${ }^{e}$ & $3.8(7)$ & $1.5(2)$ & $9.3(5)$ & $f$ & .023 \\
\hline $\begin{array}{l}\text { At least one contact during the } \\
\text { program, } \%(\mathrm{~N})\end{array}$ & $97.7(169)$ & $96.7(118)$ & $100(51)$ & $\mathrm{f}$ & .321 \\
\hline $\begin{array}{l}\text { Lost contact temporarily (> } 2 \\
\text { month), \% (N) }\end{array}$ & $22.8(38)$ & $20.3(24)$ & $28.6(14)$ & $\chi^{2}(1)=1.335$ & 248 \\
\hline Lost contact definitively, \% (N) & $13.9(23)$ & $12.8(15)$ & $16.3(8)$ & $\chi^{2}(1)=0.356$ & .551 \\
\hline
\end{tabular}

\footnotetext{
Note. $\mathrm{a}=$ Based on Modified Vocational Status Index (MVSI). $\mathrm{b}=$ Based on Modified Location Code Index Independent living (MLCl). $\mathrm{c}=$ Complete insight. $d=$ Moderate to severe substance use. $e=$ any other non prescribed drugs and narcotics. $f=$ Fisher Exact Test. PANSS: Positive and Negative Syndrome Scale. MADRS: Montgomery Asberg Depression Rating Scale. YMRS: Young Mania Rating Scale. CMRS: case manager rating scale.
} 